\title{
Polarization squeezing and nonclassical properties of light
}

\author{
Alfredo Luis \\ Departamento de Óptica, Facultad de Ciencias Físicas, Universidad Complutense, 28040 Madrid, Spain \\ Natalia Korolkova \\ School of Physics and Astronomy, University of St. Andrews, North Haugh, St. Andrews KY16 9SS, United Kingdom
}

(Received 14 July 2006; published 23 October 2006)

\begin{abstract}
We formulate polarization uncertainty relations and polarization squeezing criteria in a $\mathrm{SU}(2)$ invariant manner. In this formulation the $\mathrm{SU}(2)$ coherent states are the only minimum uncertainty states. We show that polarization squeezing is a nonclassical property. We analyze the relation between polarization squeezing and other nonclassical properties such as entanglement and quadrature squeezing.
\end{abstract}

DOI: 10.1103/PhysRevA.74.043817

PACS number(s): 42.50.Dv, 03.65.Ca, 42.25.Ja

\section{INTRODUCTION}

Squeezing of quantum uncertainty in some variable is recognized as a distinctive nonclassical signature. This is clearly illustrated by quadrature squeezing. It was first introduced in the context of high-precision measurements and in the last decade is extensively exploited in the rapidly developing field of quantum information processing [1]. The concept of squeezing can be translated as well to other variables such as polarization of light.

In such a case the intuitive idea of squeezing implies that polarization fluctuations are reduced below some prescribed level. In this regard, polarization is a more complex variable than quadrature, so it turns out that for polarization it is necessary to explicitly specify what is understood by polarization fluctuations. In other words, while for quadratures the choice of a coherent, minimum uncertainty state as the reference level is clearly singled out, there seem to be no such universal reference state for quantum polarization since coherent and minimum uncertainty states may not necessarily coincide for polarization variables [2]. Unlike quadrature squeezing it appears that the precise quantitative formulation of polarization squeezing depends on the purpose, i.e., on the envisaged application of polarization squeezed light. All this means is that there is no unique criterion for polarization squeezing. Different possibilities are examined in Sec. II.

However, there are some general requirements to a physically sensible squeezing criterion. For example, quadrature squeezing is knowingly invariant under linear unitary local transformations. In this paper we discuss the idea of the $\mathrm{SU}(2)$ invariance and suggest that the $\mathrm{SU}(2)$ invariance should be an important property of a measure for polarization squeezing. Furthermore, in Sec. II we show that it defines suitable polarization operators that greatly simplify the description of polarization fluctuations, uncertainty relations, and criteria for polarization squeezing.

In principle the reduction of polarization fluctuations below some prescribed level does not necessarily imply nonclassical behavior since a distinctive feature of classical light is the possibility of having precise nonfluctuating values of all optical variables simultaneously, including polarization. We show that polarization squeezing is actually a nonclassical property (Sec. III). Then we examine whether there is any logical relation between polarization squeezing and other nonclassical properties (Secs. IV and V). That is, we investigate whether polarization squeezing is a specific nonclassical property or, on the contrary, is a byproduct of other nonclassical features such as quadrature squeezing or entanglement, for instance.

\section{POLARIZATION SQUEEZING}

The concept of polarization squeezing implies polarization fluctuations reduced below some prescribed level. However, contrary to the case of quadrature squeezing, there is no unique squeezing criterion here: Polarization fluctuations can be assessed in different ways for different purposes. The most widely used criteria of polarization squeezing are examined in Sec. II C. Before doing this, in Secs. II A and II B we review some basic concepts required to properly formulate these criteria.

\section{A. Stokes operators and reference states}

Polarization state and polarization fluctuations can be conveniently described in terms of the Stokes operators

$$
\begin{gathered}
S_{0}=a_{1}^{\dagger} a_{1}+a_{2}^{\dagger} a_{2}, \quad S_{x}=a_{1}^{\dagger} a_{2}+a_{2}^{\dagger} a_{1}, \\
S_{y}=i\left(a_{2}^{\dagger} a_{1}-a_{1}^{\dagger} a_{2}\right), \quad S_{z}=a_{1}^{\dagger} a_{1}-a_{2}^{\dagger} a_{2},
\end{gathered}
$$

satisfying the $\mathrm{SU}(2)$-like commutation relations $\left[S_{x}, S_{y}\right]$ $=2 i S_{z}$ (and cyclic permutations) and $\left[S_{0}, \boldsymbol{S}\right]=\mathbf{0}$, where $a_{1}, a_{2}$ are the bosonic annihilation operators of a two-mode field. We can regard $S_{x}, S_{y}, S_{z}$ as projections along Cartesian axes of a Stokes vector of operators $S=\left(S_{x}, S_{y}, S_{z}\right)$, while $S_{0}$ represents the total number of photons, and the length of the Stokes vector is given by $S^{2}=S_{0}\left(S_{0}+2\right)$.

The polarization state is usually specified by the Stokes parameters $\langle\boldsymbol{S}\rangle$ which are the mean values of the Stokes operators. Likewise, polarization fluctuations can be expressed in terms of the variances $\left(\Delta S_{j}\right)^{2}, j=x, y, z$, of the Stokes operators. These variances are not independent since they must satisfy the three standard uncertainty relations of an angular momentum

$$
\Delta S_{x} \Delta S_{y} \geqslant\left|\left\langle S_{z}\right\rangle\right|, \quad \Delta S_{x} \Delta S_{z} \geqslant\left|\left\langle S_{y}\right\rangle\right|, \quad \Delta S_{y} \Delta S_{z} \geqslant\left|\left\langle S_{x}\right\rangle\right| .
$$


The critical level of fluctuations for polarization squeezing is usually established by coherent states, either quadrature coherent states $\left|\alpha_{1}, \alpha_{2}\right\rangle$ or $\mathrm{SU}(2)$ coherent states $|n, \theta, \phi\rangle$. They are closely related since

$$
\left|\alpha_{1}, \alpha_{2}\right\rangle=e^{-r^{2} / 2} \sum_{n=0}^{\infty} \frac{r^{n} e^{i n \delta}}{\sqrt{n !}}|n, \theta, \phi\rangle
$$

with

$$
|n, \theta, \phi\rangle=\sum_{m=0}^{n}\left(\begin{array}{c}
n \\
m
\end{array}\right)^{1 / 2}\left(\cos \frac{\theta}{2}\right)^{n-m}\left(\sin \frac{\theta}{2}\right)^{m} e^{-i m \phi}|m\rangle_{1}|n-m\rangle_{2} .
$$

Here $|m\rangle_{1},|n-m\rangle_{2}$ are number states in the corresponding modes. The above state parameters $\alpha_{1,2}$ are connected by the relations

$$
\alpha_{1}=r \sin \frac{\theta}{2} e^{i \delta} e^{-i \phi}, \quad \alpha_{2}=r \cos \frac{\theta}{2} e^{i \delta},
$$

where $n$ represents the total number of photons, and it holds that $S_{0}|n, \theta, \phi\rangle=n|n, \theta, \phi\rangle$.

Concerning polarization fluctuations, for the quadrature coherent states $\left|\alpha_{1}, \alpha_{2}\right\rangle$ we have

$$
\left(\Delta S_{x}\right)^{2}=\left(\Delta S_{y}\right)^{2}=\left(\Delta S_{z}\right)^{2}=\left(\Delta S_{0}\right)^{2}=\left\langle S_{0}\right\rangle,
$$

while for the $\mathrm{SU}(2)$ coherent states $|n, \theta, \phi\rangle$

$$
\Delta S_{0}=0, \quad\left(\Delta S_{j}\right)^{2}=n-\frac{1}{n}\left\langle S_{j}\right\rangle^{2},
$$

for $j=x, y, z$.

\section{B. $\mathbf{S U}(2)$ invariance}

Next we argue that criteria for polarization squeezing should be invariant under SU(2) transformations. It means, they should be invariant under the action of linear energy conserving transformations of the complex amplitudes $a_{1}, a_{2}$, such as the ones produced by beam splitters and phase plates. This is because they are rotations of the Poincare sphere which purely displace the polarization distribution, i.e., change the Stokes parameters but preserve the form of the polarization distribution. Since fluctuations depend exclusively on the form of the corresponding probability distribution irrespective of its location we conclude that any criterion of polarization squeezing should be invariant under SU(2) transformations.

The idea of invariance suggests that in general we should avoid formulating squeezing criteria in terms of the components of $\boldsymbol{S}$ along arbitrary unspecified Cartesian axes without further caution.

A simple procedure to guarantee $\mathrm{SU}(2)$ invariance is obtained by using specific components of the Stokes operators referred to the mean value $\langle\boldsymbol{S}\rangle$. These are the parallel component $S_{\|}$along $\langle\boldsymbol{S}\rangle$, with $\left|\left\langle S_{\|}\right\rangle\right|=|\langle\boldsymbol{S}\rangle|$, and two components orthogonal to $\langle\boldsymbol{S}\rangle$, denoted by $S_{\perp}$, with $\left\langle S_{\perp}\right\rangle=0$. Two orthogonal components $S_{\perp 1}, S_{\perp 2}$ define the so-called dark plane (see also Ref. [3]).
Actually, in many works on polarization squeezing the invariance requirement is implicitly taken into account. This occurs when the Cartesian axes are chosen so that $\langle\boldsymbol{S}\rangle$ is aligned with one of the $S_{j}$ components, say $S_{x}$, that plays the role of $S_{\|}$, while $S_{z}, S_{y}$ lie in the plane orthogonal to $\langle\boldsymbol{S}\rangle$ playing the roles of $S_{\perp 1}, S_{\perp 2}$.

Thus, the use of the operator basis $S_{\|}, S_{\perp 1,2}$ guarantees the $\mathrm{SU}(2)$ invariance of the polarization squeezing criterion. A further advantage of using this operator basis is that it allows us to reduce the three uncertainty relations (2.2) for the Stokes operators to a single nontrivial one:

$$
\Delta S_{\perp 1} \Delta S_{\perp 2} \geqslant\left|\left\langle S_{\|}\right\rangle\right| .
$$

The other two become trivial

$$
\Delta S_{\perp 1} \Delta S_{\|} \geqslant 0, \quad \Delta S_{\perp 2} \Delta S_{\|} \geqslant 0 .
$$

The more complex situation with defining polarization squeezing largely stems from the discrepancy between coherent and minimum uncertainty states for the Stokes operators. This is unlike quadrature coherent states which are minimum uncertainty states. Importantly, when uncertainty relations are expressed in the form of Eqs. (2.8) and (2.9) both quadrature coherent states and $\mathrm{SU}(2)$ coherent states are minimum uncertainty states in the sense of leading to an equality in Eq. (2.8), since for these states we have

$$
\left(\Delta S_{\perp 1}\right)^{2}=\left(\Delta S_{\perp 2}\right)^{2}=\left|\left\langle S_{\|}\right\rangle\right|=\left\langle S_{0}\right\rangle .
$$

On the other hand, the $\mathrm{SU}(2)$ coherent states are the only states satisfying the three equalities in Eqs. (2.8) and (2.9) simultaneously, as demonstrated in the Appendix, while quadrature coherent states only satisfy the equality in Eq. (2.8), since for them $\Delta S_{\|} \neq 0$ and this precludes the fulfillment of the equalities in Eq. (2.9). It is worth noting that the minimum uncertainty character of $\mathrm{SU}(2)$ coherent states no longer holds for arbitrary Cartesian projections of $S$. Other measures of polarization fluctuations for which SU(2) coherent states are the only minimum uncertainty states are shown in Ref. [4].

The above choice of polarization operators $S_{\perp}, S_{\|}$defines specific field modes with complex amplitude operators $b_{1}, b_{2}$ that allow one to express $S_{\|}$as follows:

$$
S_{\|}=b_{1}^{\dagger} b_{1}-b_{2}^{\dagger} b_{2},
$$

with $\left\langle b_{1}^{\dagger} b_{2}\right\rangle=0$. In this mode basis, the $\mathrm{SU}(2)$ coherent states factorize as the product of a number state in one mode and vacuum state in the other mode, $|n, \theta, \phi\rangle_{a}=|n\rangle_{b 1}|0\rangle_{b 2}$ or $|n, \theta, \phi\rangle_{a}=|0\rangle_{b 1}|n\rangle_{b 2}$. For the rest of the mode bases the $\mathrm{SU}(2)$ coherent states are entangled states of the constituting modes. This is consistent with the fact that for ensembles of spin-1/2 systems (such as two-level atoms) the collective $\mathrm{SU}(2)$ coherent states factorize as the product of identical single particle states. In our case we have that all the photons are in the same mode.

\section{Polarization squeezing criteria}

Several definitions of polarization squeezing can be found in the literature. The simpler one focuses directly on the 
comparison of the fluctuations of the orthogonal components $\Delta S_{\perp}$ with the fluctuations of the same components for quadrature or $\mathrm{SU}(2)$ coherent states, for which $\Delta S_{\perp}=\sqrt{\left\langle S_{0}\right\rangle}$ for every $S_{\perp}$. Therefore, polarization squeezing occurs if there are orthogonal components with $[5,6]$

$$
\Delta S_{\perp}<\sqrt{\left\langle S_{0}\right\rangle} .
$$

We will see in the next section that this criterion admits a simple expression in terms of complex amplitude operators.

Another criterion based on precision measurements defines polarization squeezing whenever for a suitable orthogonal component $S_{\perp}$ it occurs that $[6,7]$

$$
\frac{\Delta S_{\perp}}{\mid\left\langle S_{\|}\right\rangle}<\frac{1}{\sqrt{\left\langle S_{0}\right\rangle}} .
$$

The states fulfilling this criterion allow one to perform measurements with a precision beyond the level achievable when using quadrature coherent states or $\mathrm{SU}(2)$ coherent states (that lead to the equality in the preceding relation). This can be also regarded as the SU(2) invariant form of another measure of spin squeezing or entanglement of spin-1/2 particles expressed in Cartesian components as $[8,9]$

$$
\frac{\left(\Delta S_{z}\right)^{2}}{\left\langle S_{x}\right\rangle^{2}+\left\langle S_{y}\right\rangle^{2}}<\frac{1}{\left\langle S_{0}\right\rangle} \text {. }
$$

Finally we can consider a suitable analogy with quadrature squeezing derived from the uncertainty relation (2.8) by stating that there is polarization squeezing provided that $[2,10]$

$$
\left(\Delta S_{\perp 1}\right)^{2}<\left|\left\langle S_{\|}\right\rangle\right|<\left(\Delta S_{\perp 2}\right)^{2},
$$

for a suitable pair of components $S_{\perp j}$ in the dark plane. Equivalently, if Eq. (2.15) holds then for a suitable orthogonal component $S_{\perp}=S_{\perp 1}$ we have

$$
\frac{\left(\Delta S_{\perp}\right)^{2}}{\left|\left\langle S_{\|}\right\rangle\right|}<1
$$

Vice versa, if Eq. (2.16) holds for a component $S_{\perp 1}=S_{\perp}$ then from Eqs. (2.8) and (2.16) there is another component $S_{\perp 2}$ with $\left.\left(\Delta S_{\perp 2}\right)^{2} \geqslant\left|\left\langle S_{\|}\right\rangle\right|^{2} /\left(\Delta S_{\perp 1}\right)^{2}\right\rangle\left|\left\langle S_{\|}\right\rangle\right|$so that both inequalities in Eq. (2.15) are satisfied simultaneously. This criterion (2.16) might be useful for quantum information applications of polarization squeezing, e.g., for polarization entanglement generation, since the relevant entanglement criteria deal with squeezing properties derived from uncertainty relations for sums and differences of single party observables.

Although these three criteria are not equivalent, there is a definite relationship between them since they can be definitely arranged in a series of increasing stringency. Since it always holds that $\left\langle S_{0}\right\rangle \geqslant\left|\left\langle S_{\|}\right\rangle\right|$, the fulfillment of criterion (2.13) implies that criterion (2.16) is satisfied, which in turn implies that criterion (2.12) holds,

$$
\left(\Delta S_{\perp}\right)^{2}<\frac{\left\langle S_{\|}\right\rangle^{2}}{\left\langle S_{0}\right\rangle} \leqslant\left|\left\langle S_{\|}\right\rangle\right| \leqslant\left\langle S_{0}\right\rangle,
$$

while the converse are not necessarily true. Thus the weakest criterion (2.12) is a necessary condition for polarization squeezing since this is satisfied whenever there is polarization squeezing according to any one of the other stronger criteria. In other words, all polarization squeezed states satisfy criterion (2.12).

It can be appreciated that in these criteria the fluctuations of $S_{\|}$are absent. It seems that the only way to include $\Delta S_{\|}$ should be by adding a condition similar to Eq. (2.12) in the form $\Delta S_{\|}<\sqrt{\left\langle S_{0}\right\rangle}$. In such a case it should be understood that for this last expression the reference level for polarization fluctuations would be quadrature coherent states exclusively, since for $\mathrm{SU}(2)$ coherent states $\Delta S_{\|}=0$. The inclusion of $\Delta S_{\|}$ in other criteria of the form (2.13), (2.16) is excluded from the very beginning. On the one hand, in precision measurements the fluctuations of the component $S_{\|}$play no role since only orthogonal components are measured. Moreover, the only uncertainty relations involving $\Delta S_{\|}$are trivial as shown in Eqs. (2.8), (2.9).

\section{POLARIZATION SQUEEZING AND NONCLASSICALITY}

In this section we show that polarization squeezing is always a nonclassical property. For this purpose, we express the necessary condition for polarization squeezing (2.12) in terms of complex amplitude operators because this will greatly simplify further analysis. To this end we consider the privileged mode bases defined by Eq. (2.11) so that any orthogonal component is of the form

$$
S_{\perp}=e^{-i \phi} b_{1}^{\dagger} b_{2}+e^{i \phi} b_{2}^{\dagger} b_{1},
$$

where $\phi$ is an arbitrary phase. Then we have

$$
S_{\perp}^{2}=2 b_{1}^{\dagger} b_{1} b_{2}^{\dagger} b_{2}+b_{1}^{\dagger} b_{1}+b_{2}^{\dagger} b_{2}+e^{-2 i \phi} b_{1}^{\dagger 2} b_{2}^{2}+e^{2 i \phi} b_{2}^{\dagger 2} b_{1}^{2},
$$

and the condition (2.12)

$$
\left(\Delta S_{\perp}\right)^{2}=\left\langle S_{\perp}^{2}\right\rangle\left\langle\left\langle S_{0}\right\rangle=\left\langle b_{1}^{\dagger} b_{1}\right\rangle+\left\langle b_{2}^{\dagger} b_{2}\right\rangle,\right.
$$

for suitable $\phi$ is equivalent to

$$
\left\langle b_{1}^{\dagger} b_{1} b_{2}^{\dagger} b_{2}\right\rangle<\left|\left\langle b_{1}^{\dagger 2} b_{2}^{2}\right\rangle\right|, \quad\left\langle b_{1}^{\dagger} b_{2}\right\rangle=0 .
$$

where the second relation ensures that $\left\langle S_{\perp}\right\rangle=0$ for all $S_{\perp}$.

It can be seen that the first relation in Eq. (3.4) becomes an equality for quadrature coherent states as well as for $\mathrm{SU}(2)$ coherent states, even when $\left\langle b_{1}^{\dagger} b_{2}\right\rangle \neq 0$.

As a simple consequence we get that the fulfillment of condition (3.4) implies that polarization squeezing is a nonclassical effect since it demands negative values for the $P\left(\beta_{1}, \beta_{2}\right)$ quasiprobability distribution associated to normal ordering,

$$
\rho=\int d^{2} \beta_{1} d^{2} \beta_{2} P\left(\beta_{1}, \beta_{2}\right)\left|\beta_{1}, \beta_{2}\right\rangle\left\langle\beta_{1}, \beta_{2}\right|,
$$

where $\rho$ is the density matrix and $\left|\beta_{1}, \beta_{2}\right\rangle$ are quadrature coherent states in modes $b_{1}, b_{2}$. This is because Eq. (3.4) can be expressed as follows: 


$$
\begin{aligned}
& \int d^{2} \beta_{1} d^{2} \beta_{2} P\left(\beta_{1}, \beta_{2}\right)\left|\beta_{1} \beta_{2}\right|^{2} \\
& \quad<\left|\int d^{2} \beta_{1} d^{2} \beta_{2} P\left(\beta_{1}, \beta_{2}\right) \beta_{1}^{* 2} \beta_{2}^{2}\right|,
\end{aligned}
$$

whereas for classical distributions [i.e., positive $P\left(\beta_{1}, \beta_{2}\right)$ ] the opposite relation always holds

$$
\begin{aligned}
& \left|\int d^{2} \beta_{1} d^{2} \beta_{2} P\left(\beta_{1}, \beta_{2}\right) \beta_{1}^{* 2} \beta_{2}^{2}\right| \\
& \quad \leqslant \int d^{2} \beta_{1} d^{2} \beta_{2} P\left(\beta_{1}, \beta_{2}\right)\left|\beta_{1} \beta_{2}\right|^{2} .
\end{aligned}
$$

\section{POLARIZATION SQUEEZING AND ENTANGLEMENT}

Once we have proven that polarization squeezing is a nonclassical effect we next examine whether there is any definite relation with other nonclassical properties. In this section we examine its relation with entanglement.

To this end it is necessary to specify once for all the mode bases where the entanglement will be examined. This is because different mode bases are related by SU(2) transformations that affect the amount of entanglement. In this context we think that the most appropriate mode bases are the ones defined by relation (2.11).

After this mode choice we can prove that entanglement in modes $b_{1}, b_{2}$ is a necessary condition for polarization squeezing, which is the natural counterpart for light fields of the equivalent result already known for ensembles of spin systems $[5,8]$. We begin by noting that the following decomposition of the system density matrix $\rho$ is useful for the computation of polarization properties:

$$
\rho=\sum_{n=0}^{\infty} p_{n} \rho_{n}+(\text { crossed terms }),
$$

where the states $\rho_{n}$ are the projections of $\rho$ on the subspaces of fixed total photon number, and "crossed terms" refers to matrix elements connecting subspaces of different total photon number. This decomposition is particularly useful because the commutation relation $\left[S_{0}, S\right]=\mathbf{0}$ implies that for any polarization property $A$ it holds that $\left[S_{0}, A\right]=0$ so that the "crossed terms" do not contribute to the mean value of $A$ which reduces to the weighted sum of the mean values of $A$ in the states $\rho_{n}$,

$$
\langle A\rangle=\operatorname{tr}(A \rho)=\sum_{n=0}^{\infty} p_{n} \operatorname{tr}\left(A \rho_{n}\right)=\sum_{n=0}^{\infty} p_{n}\langle A\rangle_{n},
$$

where the subscript $n$ denotes the mean value in the state $\rho_{n}$.

Next we can demonstrate that the global state $\rho$ satisfies criterion (3.4) only if it satisfied for at least one $\rho_{n}$. Otherwise, if no $\rho_{n}$ satisfies it, we have $\left\langle b_{1}^{\dagger} b_{1} b_{2}^{\dagger} b_{2}\right\rangle_{n} \geqslant\left|\left\langle b_{1}^{\dagger 2} b_{2}^{2}\right\rangle_{n}\right|$ for all $n$ and then

$$
\begin{aligned}
\left\langle b_{1}^{\dagger} b_{1} b_{2}^{\dagger} b_{2}\right\rangle & =\sum_{n=0}^{\infty} p_{n}\left\langle b_{1}^{\dagger} b_{1} b_{2}^{\dagger} b_{2}\right\rangle_{n} \geqslant \sum_{n=0}^{\infty} p_{n}\left|\left\langle b_{1}^{\dagger 2} b_{2}^{2}\right\rangle_{n}\right| \\
& \geqslant\left|\sum_{n=0}^{\infty} p_{n}\left\langle b_{1}^{\dagger 2} b_{2}^{2}\right\rangle_{n}\right|=\left|\left\langle b_{1}^{\dagger 2} b_{2}^{2}\right\rangle\right|,
\end{aligned}
$$

so that $\rho$ is not polarization squeezed.

Finally, we demonstrate the desired relation between polarization squeezing and entanglement by showing that for all separable states $\rho_{n}^{\text {sep }}$ we get $\left\langle b_{1}^{\dagger} b_{1} b_{2}^{\dagger} b_{2}\right\rangle_{n} \geqslant\left|\left\langle b_{1}^{\dagger 2} b_{2}^{2}\right\rangle_{n}\right|$. This is because the most general separable state with a fixed total number of photons $n$ in modes $b_{1}, b_{2}$ is necessarily of the form

$$
\rho_{n}^{\mathrm{sep}}=\sum_{m=0}^{n} q_{m}|m\rangle_{1}\langle m|\otimes| n-m\rangle_{2}\langle n-m|,
$$

where $|m\rangle_{1},|n-m\rangle_{2}$ are number states in modes $b_{1}, b_{2}$. This form implies that $\left\langle b_{1}^{\dagger 2} b_{2}^{2}\right\rangle=0$ so that $\left\langle b_{1}^{\dagger} b_{1} b_{2}^{\dagger} b_{2}\right\rangle_{n} \geqslant\left|\left\langle b_{1}^{\dagger 2} b_{2}^{2}\right\rangle_{n}\right|$.

Therefore, if all projections $\rho_{n}$ are separable then $\rho$ is not polarization squeezed. In other words, a necessary condition for polarization squeezing is that at least one projection $\rho_{n}$ must be entangled.

It is worth stressing that the existence of entangled projections $\rho_{n}$ is independent of whether the total state $\rho$ is entangled or not. For example, the product states $\left|\psi_{1}\right\rangle\left|\psi_{2}\right\rangle$ have entangled projections $\rho_{n}$ provided that $\left|\psi_{j}\right\rangle$ are not number states.

It must be noted that in general there is no direct relationship between the amount of entanglement and amount of polarization squeezing as illustrated by the following example. Let us consider pure two-photon states expressed in the photon number basis in modes $b_{1}, b_{2}$ as follows:

$$
\alpha|2\rangle_{1}|0\rangle_{2}+\beta|1\rangle_{1}|1\rangle_{2}+\gamma|0\rangle_{1}|2\rangle_{2} .
$$

In this case the condition $\left\langle b_{1}^{\dagger} b_{2}\right\rangle=0$ is satisfied by two different sets of states (i) $\beta=0$ or (ii) $|\alpha|=|\gamma|$.

In both cases we have

$$
\left\langle S_{\|}\right\rangle=2\left(|\alpha|^{2}-|\gamma|^{2}\right), \quad\left(\Delta S_{\perp}\right)_{\min }^{2}=2\left(|\beta|^{2}+1-2|\alpha \gamma|\right) .
$$

It can be easily seen using any criterion (2.12), (2.13), or (2.15), that the maximum polarization squeezing (defined as the maximum distance between the left and right sides of the corresponding criterion) is reached in all cases by the states with $\beta=0$ and $|\alpha|=|\gamma|$ [i.e., the intersection of the two sets (i) and (ii)].

Therefore, maximum polarization squeezing is not equivalent to maximum entanglement in modes $b_{1}, b_{2}$ since the states of maximum polarization squeezing are not maximally entangled states according to basic entanglement measures because of the lack of the component $|1,1\rangle$.

\section{POLARIZATION SQUEEZING AND QUADRATURE SQUEEZING}

Next we examine whether polarization squeezing is equivalent to quadrature squeezing or, on the contrary, they 
are independent concepts. We begin by examining this issue for different categories of field states. We show that in many situations, including those of most practical relevance, polarization squeezing is equivalent to quadrature squeezing. However, this equivalence is not universally valid since there are quadrature squeezed states without polarization squeezing [11] and polarization squeezed states without quadrature squeezing.

\section{A. Separable factorized states}

For factorized states $\rho=\rho_{1} \otimes \rho_{2}$ in modes $b_{1}, b_{2}$ defined by Eq. (2.11) polarization squeezing is always equivalent to quadrature squeezing. Assuming factorization, the condition (3.4) becomes

$$
\left\langle b^{\dagger} b\right\rangle<\left|\left\langle b^{2}\right\rangle\right|,
$$

for at least one of the modes $b_{1}, b_{2}$. We define the $X, Y$ quadratures of the mode $b, b=(X+i Y) / \sqrt{2}$, such that $\langle(X Y$ $+Y X)\rangle=0$ and Eq. (5.1) becomes

$$
\left\langle X^{2}\right\rangle+\left\langle Y^{2}\right\rangle-1<\left|\left\langle Y^{2}\right\rangle-\left\langle X^{2}\right\rangle\right|
$$

Assuming for definiteness and without loss of generality that $\left\langle X^{2}\right\rangle<\left\langle Y^{2}\right\rangle$ we get that (5.2) implies $\left\langle X^{2}\right\rangle<1 / 2$ and therefore $(\Delta X)^{2}<1 / 2$ so that mode $b$ presents quadrature squeezing.

\section{B. Separable nonfactorized states}

Concerning the case of general separable states in modes $b_{1}, b_{2}$,

$$
\rho=\sum_{j} p_{j} \rho_{1, j} \otimes \rho_{2, j},
$$

we can show that polarization squeezing arises only if at least one of the states $\rho_{1, j} \otimes \rho_{2, j}$ presents quadrature squeezing.

Following the same steps in the Eq. (4.3) of Sec. IV after replacing $n$ by $j$ and $\rho_{n}$ by $\rho_{1, j} \otimes \rho_{2, j}$ we get that the global state $\rho$ satisfies criterion (3.4) only if it satisfied for at least one $\rho_{1, j} \otimes \rho_{2, j}$. Since these are factorized states we can apply the result of the preceding subsection to conclude that polarization squeezing requires that at least one $\rho_{1, j} \otimes \rho_{2, j}$ must present quadrature squeezing.

However, it is worth stressing that the global state $\rho$ need not be quadrature squeezed. This can be demonstrated by means of a simple counterexample

$$
\rho=\frac{1}{2}\left(|0\rangle_{1}\left\langle 0\left|\otimes \rho_{2}+\right| \alpha\right\rangle_{1}\langle\alpha|\otimes| \xi\rangle_{2}\langle\xi|\right),
$$

where $|0\rangle_{2}$ is the vacuum, $|\alpha\rangle_{1}$ is a coherent state, $|\xi\rangle_{2}$ is the squeezed vacuum, and $\rho_{2}$ is an arbitrary state to be specified later. For simplicity we assume the amplitude $\alpha$ to be real and

$$
\operatorname{tr}\left(\rho_{2} b_{2}\right)=0, \quad{ }_{2}\left\langle\xi\left|\left(X_{2} Y_{2}+Y_{2} X_{2}\right)\right| \xi\right\rangle_{2}=0,
$$

where $X_{2}, Y_{2}$ are the quadratures of mode $b_{2}, b_{2}=\left(X_{2}\right.$ $\left.+i Y_{2}\right) / \sqrt{2}$, so that for the state $|\xi\rangle_{2}$ we have $\Delta X_{2}=e^{-r} / \sqrt{2}$, where $r$ is the squeezing parameter. In such a case it can be seen that for the state (5.4) we get $\left\langle b_{1}^{\dagger} b_{2}\right\rangle=0$ and

$$
\left\langle b_{1}^{\dagger} b_{1} b_{2}^{\dagger} b_{2}\right\rangle=\frac{\alpha^{2}}{2} \sinh ^{2} r, \quad\left\langle b_{1}^{\dagger 2} b_{2}^{2}\right\rangle=-\frac{\alpha^{2}}{4} \sinh (2 r),
$$

so that according to Eq. (3.4) the state (5.4) presents polarization squeezing provided that $|r|>0$, i.e., when $|\xi\rangle_{2}$ is actually a quadrature squeezed state.

Next we compute the quadrature squeezing on the global state $\rho$ by computing the uncertainty of an arbitrary quadrature

$$
X=(\cos \theta) X_{1}^{\prime}+(\sin \theta) X_{2}^{\prime},
$$

where

$$
X_{j}^{\prime}=\frac{1}{\sqrt{2}}\left(b_{j} e^{-i \delta_{j}}+b_{j}^{\dagger} e^{i \delta_{j}}\right),
$$

and $\theta, \delta_{1,2}$ are arbitrary angles. We get

$$
\begin{aligned}
(\Delta X)^{2}= & \frac{1}{2}\left\{\left(\cos ^{2} \theta\right)\left(1+\alpha^{2} \cos ^{2} \delta_{1}\right)\right. \\
& \left.+\left(\sin ^{2} \theta\right)\left[\left(\Delta X_{2}^{\prime}\right)_{\rho 2}^{2}+\left(\Delta X_{2}^{\prime}\right)_{\xi}^{2}\right]\right\},
\end{aligned}
$$

where the subscripts in $\left(\Delta X_{2}^{\prime}\right)^{2}$ indicate the state where this quantity is evaluated.

The result is that we can always choose $\rho_{2}$ with large enough $\left(\Delta X_{2}^{\prime}\right)_{\rho 2}^{2}$ so that

$$
\left(\Delta X_{2}^{\prime}\right)_{\rho 2}^{2}+\left(\Delta X_{2}^{\prime}\right)_{\xi}^{2} \geqslant 1,
$$

for every $\delta_{2}$. In such a case $\Delta X \geqslant 1 / \sqrt{2}$ and the state $\rho$ is not quadrature squeezed.

\section{Bright limit and one-mode approximation}

There is another situation where polarization squeezing is equivalent to quadrature squeezing. This occurs when the field state in one of the modes, say $a_{1}$, tends to be a classical state much more intense than the field in mode $a_{2}$, so that we can replace in Eq. (2.1) the operator $a_{1}$ by the variable $\alpha$ (assumed real for simplicity), leading to

$$
S_{x} \simeq \sqrt{2} \alpha X_{2}, \quad S_{y} \simeq \sqrt{2} \alpha Y_{2}, \quad S_{0} \simeq S_{z} \simeq \alpha^{2},
$$

where $X_{2}, Y_{2}$ are the corresponding quadratures of the mode $a_{2}$. This provides a simple one-mode approximation to polarization, which is in principle a two-mode property.

Because mode $a_{1}$ is much more intense than mode $a_{2}$ we get $S_{\|} \simeq S_{z}$ so we can take $S_{\perp}=S_{x}$ for instance, and then

$$
\Delta S_{\perp} \simeq \sqrt{2} \alpha \Delta X_{2}, \quad\left\langle S_{0}\right\rangle \simeq\left\langle S_{\|}\right\rangle \simeq \alpha^{2} .
$$

With these values all criteria of polarization squeezing (2.12), (2.13), (2.15), are equivalent to $\Delta X_{2}<1 / \sqrt{2}$, which means quadrature squeezing in mode $a_{2}$.

\section{Polarization squeezing without quadrature squeezing}

The above results suggest the equivalence between polarization squeezing and quadrature squeezing. However, next 
we show that this relation is not universal since there are polarization squeezed states without quadrature squeezing. This is the case of the state

$$
|\psi\rangle=\frac{1}{\sqrt{2}}\left(|n\rangle_{1}|n\rangle_{2}+|n+1\rangle_{1}|n-1\rangle_{2}\right),
$$

where $|n\rangle,|n \pm 1\rangle$ are the number states in modes $a_{1}, a_{2}$. In this case we have

$$
\left\langle S_{x}\right\rangle=\sqrt{n(n+1)}, \quad\left\langle S_{y}\right\rangle=0, \quad\left\langle S_{z}\right\rangle=1, \quad\left\langle S_{0}\right\rangle=2 n,
$$

so that for $n \gg 1$ we can take $\left\langle S_{\|}\right\rangle \simeq\left\langle S_{x}\right\rangle \simeq n$ and $S_{\perp} \simeq S_{z}$ for example. Since $\Delta S_{\perp} \simeq \Delta S_{z}=1$ we get that this state is polarization squeezed according to all criteria (2.12), (2.13), (2.15). In particular the strongest one (2.13) becomes

$$
\frac{\Delta S_{\perp}}{\left\langle S_{\|}\right\rangle}=\frac{1}{n}<\frac{1}{\sqrt{2 n}}
$$

which is clearly fulfilled for $n \gg 1$.

Next we examine whether the states (5.13) present quadrature squeezing for the arbitrary quadrature $X$ in Eqs. (5.7), (5.8) after performing the replacement $b_{j} \rightarrow a_{j}$. The answer is negative since

$$
(\Delta X)^{2}=\frac{1}{2}\left[2 n+1+\cos (2 \theta)+\sin (2 \theta) \cos \left(\delta_{1}-\delta_{2}\right) \sqrt{n(n+1)}\right] .
$$

For $n \gg 1$ the minimum $\Delta X$ is obtained for $\sin (2 \theta) \cos \left(\delta_{1}\right.$ $\left.-\delta_{2}\right)=-1$, leading to

$$
(\Delta X)_{\min }^{2} \simeq \frac{n}{2} \gg \frac{1}{2}
$$

so there is no quadrature squeezing in any case.

\section{CONCLUSIONS}

We have shown the advantages of a proper choice of the components of the Stokes vector expressing polarization properties. This allows one to formulate criteria for polarization squeezing in a $\mathrm{SU}(2)$ invariant way, which is, we suggest, required for a sensible definition of polarization squeezing. This also allows one to reduce the three standard uncertainty relations for the Stokes operators to a single nontrivial relation and two trivial ones. We have demonstrated that the $\mathrm{SU}(2)$ coherent states are the ones that satisfy the three uncertainty relations, while quadrature coherent states satisfy only the nontrivial one. This definition of polarization components also provides a mode basis relevant for examining the relation between polarization squeezing and mode entanglement.

Furthermore, we have proven that polarization squeezing is always a nonclassical property. When examining its relation with other nonclassical properties we have shown that entanglement is a necessary condition for polarization squeezing. In many situations (including those of most practical relevance) polarization squeezing is equivalent to quadrature squeezing. However, this relation is not universally valid since we have shown that there are polarization squeezed states without quadrature squeezing.

\section{ACKNOWLEDGMENTS}

A.L. acknowledges support from Project No. FIS200401814 of the Spanish Dirección General de Investigación del Ministerio de Educación y Ciencia. N.K. acknowledges funding from the EU Project No. FP6-511004 COVAQIAL.

\section{APPENDIX: SU(2) COHERENT STATES AS MINIMUM UNCERTAINTY STATES}

We demonstrate that the $\mathrm{SU}(2)$ coherent states are the minimum uncertainty states for the Stokes operators in the sense that they are the only states satisfying the equality both in Eqs. (2.8) and (2.9). In the photon number basis associated to the modes $b_{1}, b_{2}$, the states fulfilling the equality in the two relations in Eq. (2.9) (i.e., $\Delta S_{\|}=0$ ) are necessarily of the form

$$
|\psi\rangle=\sum_{n=0}^{\infty} c_{n}|n+m\rangle_{1}|n\rangle_{2}
$$

where we assume without loss of generality that $m \geqslant 0$.

For the state (A1) it holds that $\left\langle b_{1}^{\dagger} b_{2}\right\rangle=\left\langle b_{1}^{\dagger} b_{2}^{2}\right\rangle=0$. Then, using Eq. (3.2) for $S_{\perp}^{2}$ we get that for any $S_{\perp}$,

$$
\left(\Delta S_{\perp}\right)^{2}=m+2 \sum_{n=0}^{\infty}\left[n^{2}+n(m+1)\right]\left|c_{n}\right|^{2}, \quad\left\langle S_{\|}\right\rangle=m .
$$

The equality in Eq. (2.8) implies that $\left(\Delta S_{\perp}\right)^{2}=m$ which in turn implies $c_{n}=0$ for all $n \neq 0$ so that $|\psi\rangle=|m\rangle_{1}|0\rangle_{2}$ are $\mathrm{SU}(2)$ coherent states.
[1] S. L. Braunstein and P. van Loock, Rev. Mod. Phys. 77, 513 (2005); Quantum Information with Continuous Variables, edited by S. L. Braunstein and A. K. Pati (Kluwer Academic Publishers, Dordrecht, The Netherlands, 2003).

[2] N. Korolkova and R. Loudon, Phys. Rev. A 71, 032343 (2005); N. Korolkova, G. Leuchs, R. Loudon, T. C. Ralph, and Ch. Silberhorn, ibid. 65, 052306 (2002).
[3] V. Josse, A. Dantan, A. Bramati, M. Pinard, E. Giacobino, J. Heersink, U. L. Andersen, O. Glöckl, and G. Leuchs, in Quantum Information with continuous variables of atoms and light, edited by N. Cerf, G. Leuchs, and E. S. Polzik (Imperial College Press, London, UK, 2006), Sec. 2.1.

[4] R. Delbourgo, J. Phys. A 10, 1837 (1977); A. Luis and L. L. Sánchez-Soto, in Progress in Optics, edited by E. Wolf 
(Elsevier, Amsterdam, 2000), Vol. 41, p. 421; S. Gnutzmann and K. Życzkowski, J. Phys. A 34, 10123 (2001); A. Luis, Phys. Rev. A 66, 013806 (2002).

[5] M. Kitagawa and M. Ueda, Phys. Rev. A 47, 5138 (1993).

[6] D. J. Wineland, J. J. Bollinger, W. M. Itano, and D. J. Heinzen, Phys. Rev. A 50, 67 (1994).

[7] M. Hillery and L. Mlodinow, Phys. Rev. A 48, 1548 (1993); C. Brif and A. Mann, ibid. 54, 4505 (1996).
[8] A. Sørensen, L.-M. Duan, J. I. Cirac, and P. Zoller, Nature (London) 409, 63 (2001).

[9] S. Raghavan, H. Pu, P. Meystre, and N. P. Bigelow, Opt. Commun. 188, 149 (2001).

[10] J. Hald, J. L. Sorensen, C. Schori, and E. S. Polzik, J. Mod. Opt. 47, 2599 (2000).

[11] J. Heersink, T. Gaber, S. Lorenz, O. Glöckl, N. Korolkova, and G. Leuchs, Phys. Rev. A 68, 013815 (2003). 